\title{
Intensive chemotherapy for non-localised Burkitt's lymphoma
}

\author{
A Al-ATtAR, J PRITCHARD, T AL-SALEEM, M AL-NAIMI, N ALASH, AND A ATTRA \\ Medical City Hospital, University of Baghdad, Iraq, and Institute for Child Health, London
}

SUMMARY Between 1982 and 1984, 24 consecutively diagnosed children from Iraq with non-localised Burkitt lymphoma (Murphy stages II, III, and IV) were eligible for treatment with a multi-drug rotating chemotherapy schedule. This schedule was intensive and included early treatment directed at the central nervous system but was of only six months' duration and fairly inexpensive compared with schedules recently advocated for use in the developed world. Some patients had 'debulking' abdominal surgery, but no radiation treatment was used. There were a number of complications related to early treatment, some of them fatal, but of 13 patients entering complete remission 12 are long term survivors who are free of disease and, hopefully, cured. These results represent a substantial improvement over our experience before $1982(6.9 \%$ survival).

A similar treatment approach might be adopted by other centres, especially those in developing countries where cancer accounts for a rising proportion of childhood death but whose resources are limited.

Although Burkitt's lymphoma ${ }^{1}$ is one of the most responsive tumours to chemotherapy, patients with advanced disease have proved difficult to cure. ${ }^{2}{ }^{3}$ Previous experience in Iraq is that the survival of patients with non-localised disease has been only $6.9 \%,{ }^{4}$ and other centres in developing countries, using non-intensive chemotherapy with or without radiotherapy, have reported similar results. ${ }^{5-7}$

The recent introduction of more intensive chemotherapy regimens has led to much improved survival for children with all categories of nonHodgkin's lymphoma, including those B cell tumours of undifferentiated histology that represent the 'sporadic' form of Burkitt's lymphoma. ${ }^{89}$ Although some authors have claimed that radiotherapy is valuable in the management of children with non-Hodgkin's lymphoma, ${ }^{910}$ two randomised studies have shown no benefit when radiotherapy was delivered to sites of previous bulky tumour, ${ }^{11} 12$ a finding that complements the clinical observation that primary site recurrence is unusual in this disease. Recurrence most commonly occurs, within six months of diagnosis, in bone marrow or the central nervous system, or both, and is then difficult to control without marrow ablative ('megatherapy') approaches. ${ }^{13} 14$
Typical and atypical Burkitt's lymphoma constitutes about one half of all childhood non-Hodgkin's lymphoma presenting to our centre (Al-Saleem T. Unpublished data). Because of dissatisfaction with our previous treatment results, we designed a protocol that incorporated (a) careful staging, (b) the use of 'normal dose' but intensively scheduled chemotherapy with rotating drug combinations, (c) early treatment directed at the central nervous system ('central nervous system prophylaxis') with intrathecal methotrexate and moderately high dose systemic methotrexate, (d) short total duration, and (e) no radiotherapy. Debulking surgery formed no part of the protocol but was carried out in a number of patients who were admitted to the surgical units of our hospital before referral to us. The whole protocol was designed to be suitable for use in our centre in which, for example, facilities for intravenous nutrition and serum methotrexate estimation are not available and to take into account the fact that, in Iraq, parental compliance with prolonged courses of treatment is poor.

The aims of the study were to assess the practicability and toxicity of the treatment regimen in a consecutive series of patients, to determine the complete response and survival rates, and to collect information on patterns of relapse. 


\section{Patients and methods}

From February 1982 to February 1984, 24 children with non-localised Burkitt's lymphoma, consecutively admitted to the paediatric oncology unit of Medical City Hospital, were entered into the study. There were 15 boys and nine girls, and their ages ranged from 2.5 to 12 years (median 4 years). Twenty three patients had had no previous treatment, and one had recurrent disease 12 months after completing six weeks of treatment with nonintensive chemotherapy (oral cyclophosphamide and methotrexate, vincristine, and intravenous cytosine arabinoside $\left.{ }^{4}\right)$. In each case the clinical diagnosis was confirmed by histological evidence of undifferentiated lymphoma or of 'L3' morphology of blasts in bone marrow. Neither immunological surface marker testing nor Epstein-Barr virus serology was available. Children with follicular centre cell or other histological subtypes of nonHodgkin's lymphoma were not included.

The following staging investigations were carried out: full blood count, bilateral bone marrow aspiration, cerebrospinal fluid cell count and cytospin, chest $x$ ray, and intravenous urogram. In addition, six non-laparotomised patients had a computed tomogram of the abdomen performed. Staging was according to Murphy's system. ${ }^{8}$

All patients were given oral or intravenous (if vomiting) allopurinol $50-100 \mathrm{mg}$ for at least 24 hours before starting chemotherapy and for one to four weeks thereafter: an attempt was made to alkalinise the urine, using intravenous sodium bicarbonate, during courses 1-5 and 8-9. Chemotherapy was given in 11 courses at one to three weekly intervals (Fig. 1), with special emphasis on the delivery of the first five courses as soon as possible after blood count recovery (neutrophils $>1 \cdot 0 \times 10^{9} / 1$ ).

Courses 1, 2, 10, and 11 consisted of 'CHOP' (for details, see Fig. 1), courses 3, 4, 5, 8, and 9 of moderate dose methotrexate (combined in courses 8 and 9 with VM 26), and courses 6 and 7 of cytosine arabinoside and 6-thioguanine. In the methotrexate courses one third of the dose was given as a bolus and the remainder as a 24 hour infusion. The first dose $(15 \mathrm{mg})$ of folinic acid 'rescue' was given intravenously at the end of the infusion of methotrexate and repeated every six hours (eight doses in all). Serum creatinine concentration was checked 48 hours from the start of treatment with methotrexate; if the concentration was $>50 \%$ higher than just before treatment folinic acid rescue was continued for five days and the next dose of methotrexate was delayed by one week. Intrathecal methotrexate was given with courses $1-6$, the dose being related to age: ${ }^{15}$ children aged $2-4$ years received $7.5 \mathrm{mg}$ per dose, while older children were given $10 \mathrm{mg}$. All treatment was stopped after course 11 , except in one child (case 19) who had blasts in the peripheral blood at diagnosis (L3 acute lymphoblastic leukaemia) and is on continuing ('maintenance') treatment (see Results). Radiotherapy

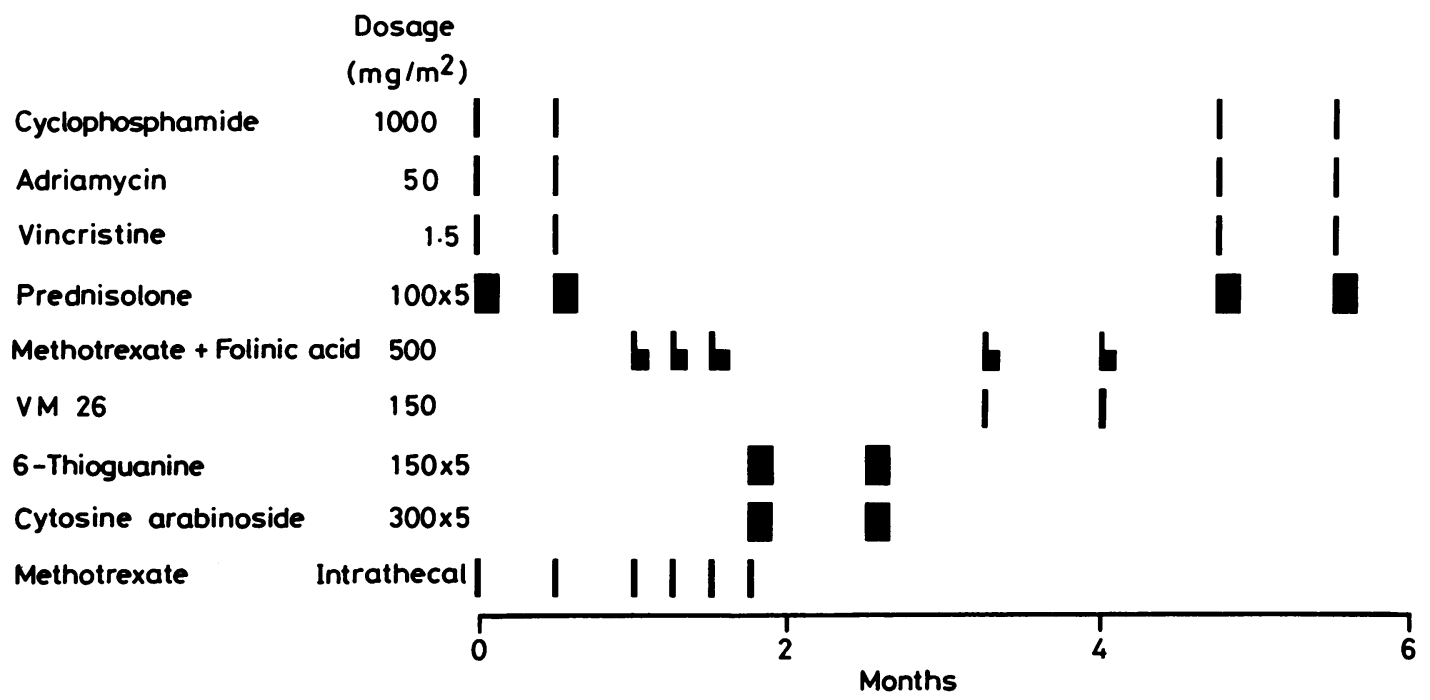

Fig 1 Diagrammatic representation of protocol. Dosage of intrathecal methotrexate was dependent on age. ${ }^{13}$ Courses $1-3$ were given as close together as blood count allowed. 
was not part of the protocol but was used as 'consolidation therapy' in one patient with testicular involvement who showed an incomplete response to chemotherapy.

Before each course the patients were examined to assess tumour response and blood count, and biochemical tests of renal and liver function were performed. Bone marrow examination was carried out every eight weeks and cerebrospinal fluid cell count and cytology after each lumbar puncture. Supportive care included intravenous fluids and antibiotics, alkalinisation of urine, and blood transfusion, as necessary. Parenteral nutrition was not available.

To determine patterns of relapse, patients who completed chemotherapy had clinical examination, bilateral bone marrow aspiration, and cerebrospinal fluid study at two monthly intervals for six months and chest $x$ ray and intravenous urogram at six months. After six months off treatment patients were monitored clinically.

\section{Results}

Diagnosis. All 24 patients fulfilled the clinical criteria for diagnosis of Burkitt's lymphoma, and the diagnosis was confirmed either cytologically (two cases) or histologically (22).
Staging and sites of disease. Five patients had stage II tumour, 12 stage III, and seven stage IV (Table 1). Eighteen paiients had extranodal abdominal disease, 13 at multiple sites. For diagnosis, four of these children underwent biopsy examinations of other disease sites (three of the jaw and one of bone marrow). In the remaining 14 , in whom disease was confined to the abdomen, laparotomy was performed. Of these 14 children, five had resection of all gross abdominal disease, but in the remainder only biopsy examination or partial resection was carried out. The incidence of involvement of the abdominal lymph nodes (five patients) was almost certainly an underestimate because laparotomies in patients with unresectable disease were not exhaustive. There were eight children with involvement of the head and neck (the jaw in seven and tonsils in one), six with involved bone marrow, and one with tumour cells in the cerebrospinal fluid. In contrast to our experience in the United Kingdom multiple simultaneous sampling of bone marrow did not, in this series, increase the yield of positive findings.

Toxicity. There was some serious toxicity related to drugs at the start of chemotherapy, with five fatalities. Two patients developed 'tumour lysis syndrome ${ }^{16}$ and died within 24 hours of receiving 'CHOP' course 1. Three others developed fever,

Table 1 Staging and sites of disease in 24 patients with Burkitt's lymphoma

\begin{tabular}{|c|c|c|c|c|c|c|}
\hline \multirow{2}{*}{$\begin{array}{l}\text { Case } \\
\text { No }\end{array}$} & \multirow[t]{2}{*}{ Stage } & \multicolumn{5}{|l|}{ Site } \\
\hline & & Abdomen & $\begin{array}{l}\text { Head and } \\
\text { and neck }\end{array}$ & $\begin{array}{l}\text { Bone } \\
\text { marrow* }\end{array}$ & $\begin{array}{l}\text { Cerebrospinal } \\
\text { fluid }\end{array}$ & $\begin{array}{l}\text { Other } \\
\text { sites }\end{array}$ \\
\hline 1 & II & $S+N$ & - & - & - & - \\
\hline 2 & III & $M+N$ & $\mathrm{~S}$ & - & - & - \\
\hline 3 & III & $\mathbf{M}$ & - & - & - & - \\
\hline 4 & III & M & - & - & - & Kidneys, nodes \\
\hline 5 & II & $\mathrm{S}+\mathrm{N}$ & - & - & - & - \\
\hline 6 & IV & - & $\mathbf{M}$ & 90 & - & Liver, spleen \\
\hline 7 & III & $\mathbf{M}$ & - & - & - & - \\
\hline 8 & III & $\mathrm{M}+\mathrm{N}$ & - & - & - & - \\
\hline 9 & III & $\mathbf{M}$ & - & - & - & $\begin{array}{l}\text { Ovaries, pouch of } \\
\text { Douglas, kidney }\end{array}$ \\
\hline 10 & II & - & $\mathbf{M}$ & - & - & - \\
\hline 11 & III & $\mathbf{M}$ & - & - & - & - \\
\hline 12 & IV & - & $\mathbf{M}$ & 30 & - & - \\
\hline 13 & III & $\mathrm{N}^{*}$ & $\mathbf{M}$ & - & - & - \\
\hline 14 & III & $\mathrm{M}$ & - & - & - & - \\
\hline 15 & IV & $\mathrm{M}$ & $\mathbf{M}$ & 90 & - & - \\
\hline 16 & IV & $\mathbf{M}$ & - & 90 & - & $\begin{array}{l}\text { Liver, spleen } \\
\text { nodes, blood }\end{array}$ \\
\hline 17 & IV & $\mathrm{S}$ & $\mathrm{S}$ & - & + & Spleen \\
\hline 18 & III & $\mathbf{M}$ & - & - & - & - \\
\hline 19 & IV & - & - & 90 & - & Blood \\
\hline 20 & II & - & $\mathrm{S}+\mathrm{N}$ & - & - & - \\
\hline 21 & III & $\mathbf{M}$ & - & - & Not done & Thyroid \\
\hline 22 & II & - & - & - & - & Testes \\
\hline 23 & III & $\mathbf{M}$ & - & - & - & - \\
\hline 24 & IV & $\mathrm{S}$ & - & 45 & - & Nodes \\
\hline
\end{tabular}

$\mathrm{S}=$ Single tumour; $\mathrm{M}=$ Multiple tumours; $\mathrm{N}=$ Regional node involvement $*=\%$ 'L3' blasts. 
jaundice, and diarrhoea while neutropenic after 'CHOP' course 1 and, although blood cultures yielded negative results, it was assumed that sepsis was the cause of death. In the surviving patients the interval between 'CHOP' courses 1 and 2 was only $2^{1 / 2-3}$ weeks and between courses 2 and 3 was only 2 weeks. After the first 'CHOP' course, treatment was well tolerated, with close adherence to the prescribed schedule and with only seven recorded episodes of toxicity, including one of neutropenia and fever, one of severe oral ulceration with raised serum creatinine concentration after treatment with methotrexate, and one of raised serum creatinine concentration alone after treatment with methotrexate. Thus only three admissions to hospital were required after a total of 144 chemotherapy courses. No adjustments to chemotherapy dose were made during the study.

It should be emphasised that some patients were in very poor general condition (Fig. 2) and that many had recently undergone major surgical proce- dures. There is little doubt that these factors contributed to the early mortality and morbidity related to chemotherapy. Surprisingly, the mean duration of symptoms, before treatment, in those patients who did not achieve complete remission was the same (four weeks) as in those patients who did. A similar observation has been made in Algerian patients. ${ }^{7}$

Response (Table 2) and survival. Two patients died and a third was lost to follow up without receiving any chemotherapy. In the remaining 21 patients chemotherapy began within one (19 children) or two weeks (two) of diagnosis. One child was lost to follow up after course 1 . Six patients succumbed to complications related to treatment, five of them after chemotherapy (see Toxicity) and one after surgery (faecal fistula with sepsis), and are grouped together as 'early deaths'. Two of these children (cases 17 and 21) died within 24 hours of starting chemotherapy, too early to assess tumour response;
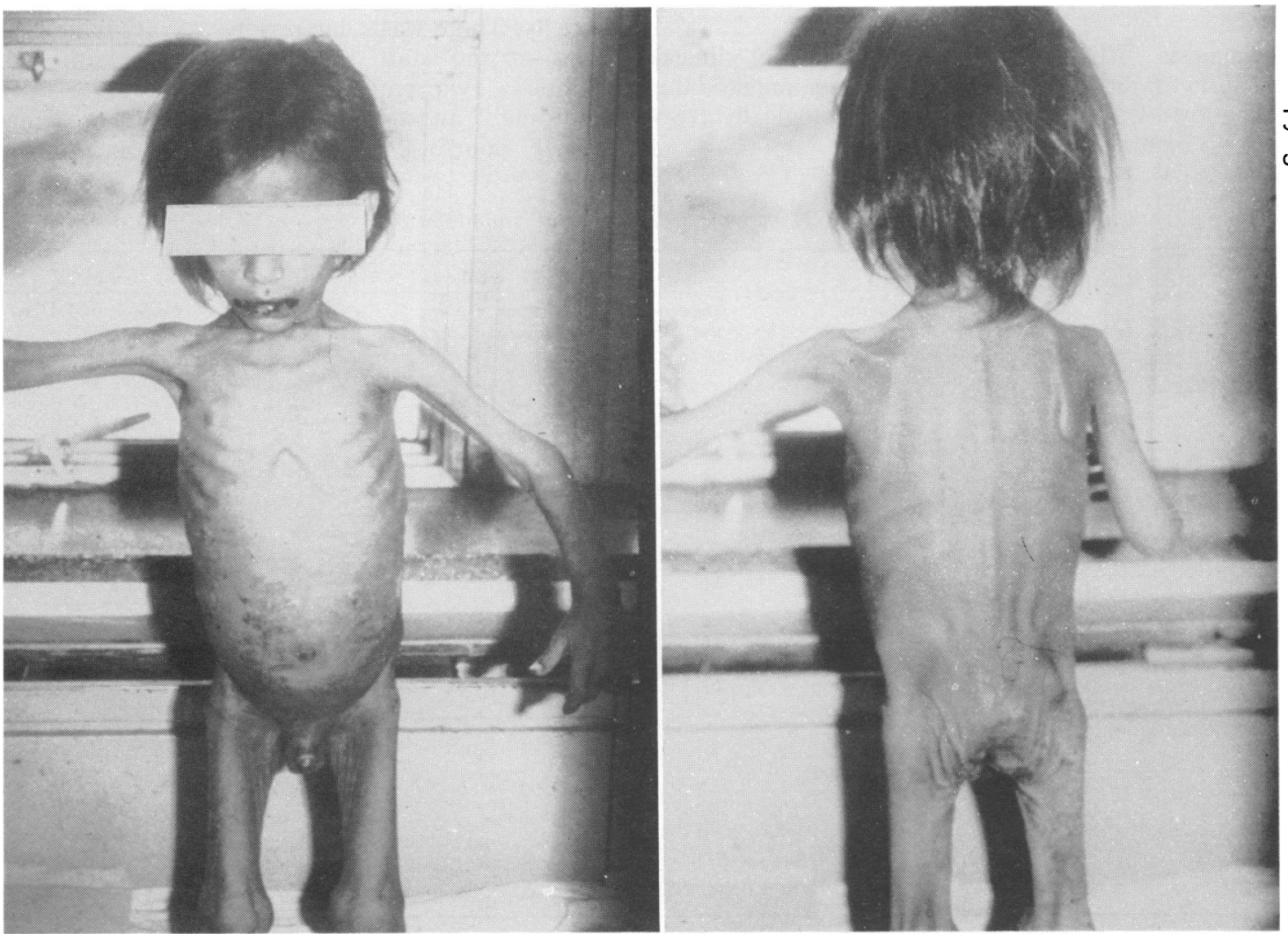

Fig. 2 Poor nutritional state of case 14 before chemotherapy. He is a long term survivor. 
Table 2 Summary of outcome of 24 patients with Burkitt's lymphoma. Numbers in parentheses indicate patients who did not receive chemotherapy

\begin{tabular}{lllllr}
\hline $\begin{array}{l}\text { State } \\
\text { stage }\end{array}$ & $\begin{array}{l}\text { Lost to } \\
\text { follow up }\end{array}$ & $\begin{array}{l}\text { Early } \\
\text { death }\end{array}$ & $\begin{array}{l}\text { Partial } \\
\text { response }\end{array}$ & $\begin{array}{l}\text { Complete } \\
\text { response }\end{array}$ & Total \\
\hline II & - & 1 & 1 & 3 & 5 \\
III & $2(1)$ & $3(1)$ & - & 7 & 12 \\
IV & - & $4(1)$ & - & 3 & 7 \\
\hline Total & 2 & 8 & 1 & 13 & 24 \\
\hline
\end{tabular}

in the other four (cases 3, 7, 16, and 24) there was clear evidence of tumour regression at the time of death seven days to two months after diagnosis.

Of the 22 patients with follow up information, 13 $(59 \%)$ achieved complete remission-three of five with stage II tumour, seven of 10 with stage III, and three of seven with stage IV. In all but two instances, complete remission was reached before chemotherapy course 3 . At diagnosis, four children had abnormal intravenous urograms, which consisted of hydronephrosis (three cases) and nonfunctioning kidney (one); all returned to normal during treatment.

The patient (case 22) who presented only with bilateral testicular involvement and showed incomplete regression of disease (partial response) was given testicular irradiation (18 Gy). Tumour cells occurred in the bone marrow and blood at four months from diagnosis, however, and he died soon afterwards.

Lymphoma recurred in only one (case 12) of the 13 patients who achieved complete response; this stage IV patient relapsed in the central nervous system and jaw and died two months later. The 12 remaining children are still in continuous complete remission eight to over 35 months (median 21 months) after diagnosis (Fig. 3). Eleven have been

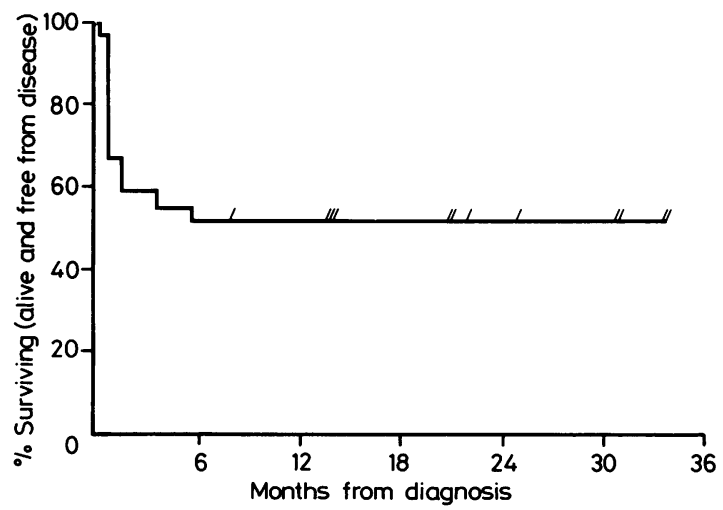

Fig. 3 Actuarial survival curve (all patients). off treatment for two to over 29 months (median 17 months). One child with L3 acute lymphoblastic leukaemia is on continuing ('maintenance') treatment with oral methotrexate/6-mercaptopurine and pulses of L-asparaginase, vincristine, prednisolone, and adriamycin.

\section{Discussion}

The design of this schedule was based on a United Kingdom Children's Cancer Study Group multidrug protocol that gave unexpectedly good results (69\% two year disease free survival) in patients with non-localised (stage II-IV) abdominal nonHodgkin's lymphoma. ${ }^{12}$ In that study the addition of radiotherapy did not improve the prognosis. In an attempt to avoid the early bone marrow and central nervous system relapse so characteristic of both endemic and non-endemic Burkitt's lymphoma, the first five courses of our protocol were administered as close together as blood counts allowed and included treatment directed at the central nervous system in the form of intrathecal and moderately high dose systemic methotrexate. Given the intensity of treatment, 'maintenance' chemotherapy was deemed unnecessary.

It has been suggested that debulking surgery reduces the relapse rate in patients with Burkitt's lymphoma,${ }^{17}$ and laparotomy is still advocated in some centres. ${ }^{18}$ In our series a higher proportion (4/5) of patients who underwent initial debulking of abdominal disease survived than of those whose tumours were considered inoperable (5/11). The group who underwent debulking, however, included two patients with stage II disease, whereas all patients in the group who did not undergo debulking had stage III or IV tumours. Although numbers are small, the long term disease free survival of these five patients who did not undergo debulking suggests that surgery does not add to chemotherapy when the regimen is of sufficient intensity. This point is of particular importance in girls because the internal genitalia (ovaries, Fallopian tubes, and uterus) are often involved by tumour: had chemotherapy been used as initial treatment in patient 9 rather than surgery, castration, with its disastrous effects on the girl's later physical and psychological development, might have been avoided.

Because 11 of our patients have now been off treatment for more than six months (median 24 months from diagnosis) we hope that most of the 12 long term survivors reported here will prove to be cured. It is especially encouraging that these results, a distinct improvement over our previous experience, have been achieved after only six months of chemotherapy and without radiotherapy. The high 
incidence of early mortality, which parallels experience in Algeria, ${ }^{7}$ might have been reduced by the availability of intravenous feeding, the avoidance of unnecessary surgical intervention before chemotherapy, and more careful monitoring during the post-chemotherapy 'tumour lysis syndrome'.

In a randomised study the United States Children's Cancer Study Group showed that a four drug chemotherapy programme ('COMP') was superior to a 10 drug schedule in the treatment of children with non-localised 'undifferentiated' non-Hodgkin lymphoma. ${ }^{18}$ Patients also received high dose (30 Gy) radiotherapy, however, to sites of bulk disease. This may have compromised the delivery of subsequent chemotherapy and so explain the greater proportion of fairly late relapses in that study compared with our own. Though two year disease free survival of the 39 patients with advanced undifferentiated lymphoma (including 29 with Burkitt's lymphoma) was only $46 \%$, Magrath et al considered that the addition of adriamycin to 'COMP'-that is, 'CHOMP'-may improve the outcome. ${ }^{19}$ Sullivan and Ramirez, by contrast, reporting a median actuarial survival of $72 \%$ in children with stage II-IV Burkitt's lymphoma treated with a regimen containing very high doses of cyclophosphamide and methotrexate, recently suggested that adriamycin offered no advantage. ${ }^{20}$

Presentation at a late stage of disease and poor compliance with treatment are facts of life in developing countries, so we thought it appropriate to include in this analysis all patients presenting to our unit during the study, including those who received no chemotherapy at all. Though disappointing at first sight, the overall actuarial survival of $55 \%$ is encouraging in this context. Even more pleasing was the low rate of relapse $(8 \%)$ among the 13 patients who achieved complete remission, suggesting that rotation of drug combinations, as in our regimen, may diminish the need for very high dose chemotherapy schedules except in patients, such as those with stage IV disease due to involvement of the central nervous system, identified as being in a very bad prognosis category. This point is of major importance for developing countries and relevant to centres in the developed world because, apart from their greater toxicity, high dose approaches require more intensive nursing and medical support than is usually available. They are also very expensive. To treat a $20 \mathrm{~kg}$ child, for example, the cost of methotrexate and folinic acid in the induction phase of the schedule of Sullivan and Ramirez is roughly $\$ 8250$ (late 1985 prices) for these drugs in the 'maintenance' phase-a total of over $\$ 16700 .{ }^{20}$ The cost of methotrexate and folinic acid in the National Cancer Institute schedule is similar. ${ }^{19}$ In our pro- tocol, by contrast, the total cost of methotrexate and folinic acid is $\$ 950$.

Nkrumah and colleagues recently reported from Ghana that 28 children with 'massive' abdominal disease had been treated with intrathecal methotrexate and, in a prospective randomised trial, with short courses either of cyclophosphamide (1400 $\mathrm{mg} / \mathrm{m}^{2}$ ) alone or intravenous cyclophosphamide $\left(700 \mathrm{mg} / \mathrm{m}^{2}\right)$, vincristine, and low dose cytosine arabinoside. ${ }^{21}$ That there was no difference in complete response rate and survival between the two randomised groups is of interest, but the most provocative feature of this small study is that the disease free survival $(58 \%)$ of patients who received cyclophosphamide alone is similar to that of our patients, treated with a more prolonged and complex regimen. If these findings are confirmed in a second Ghanaian study now in progress (Neequaye J. Personal communication) there will be a case for a randomised trial of high dose cyclophosphamide versus a more intensive multi-drug regimen, such as ours, in sporadic Burkitt's lymphoma.

Our data indicate that many patients with advanced Burkitt's lymphoma can be cured by short but intensive chemotherapy using rotating drug combinations without radiotherapy and, in at least some cases, without major surgery. Though administered without intensive nursing support and facilities such as intravenous feeding and serum methotrexate assay, treatment was associated with little morbidity except during remission induction. We believe our results have implications for other paediatric units, especially those in other developing countries where economic factors and practical considerations have hampered progress in the treatment of advanced childhood cancer.

\section{Added in proof}

More recent follow up is now available (July 1986) on the 12 patients surviving when this paper was submitted. One (case 19) relapsed 22 months from diagnosis and died two months later, but the 11 remaining children (three stage II, seven stage III, and one stage IV) are alive, well, and in complete remission 20-44 months (median 38 ) from the end of treatment.

Since the completion of this study, we have treated a further 19 patients with advanced Burkitt's lymphoma using this regimen. There were two early deaths, but 17 patients entered complete remission: 16 of these children are alive, well, and free of disease at the time of writing.

The authors gratefully acknowledge the help given by their surgical colleagues, especially Dr R Baghdadi and Dr A Al-Ramah, and the 
nursing staff, 10th floor, Medical City Hospital, in caring for these patients. We also thank Dr John Davies for independent review of the bone marrow slides, Dr T Philip for helpful comments, and Cathy Taylor and Adrianne Prior for typing the manuscript.

Dr Pritchard receives support from the Imperial Cancer Research Fund.

\section{References}

1 Burkitt DP. The discovery of Burkitt's lymphoma. Cancer 1983;51:1777-86.

2 Ziegler JL, Magrath IT, Deisseroth AB, et al. Combined modality treatment of Burkitt's lymphoma. Cancer Treat Rep 1978;62:2031-4.

${ }^{3}$ Murphy SB. Prognostic features and obstacles to cure of childhood non-Hodgkin's lymphoma. Semin Oncol 1977;4: 265-70.

4 Al-Attar A, Al-Mondhiry H, Al-Bahrani Z, et al. Burkitt's lymphoma in Iraq. Clinical and pathological study of forty-seven patients. Int J Cancer 1979;23:14-7.

5 Nkrumah FK, Perkins IV. Burkitt's lymphoma-a clinical study of 110 patients. Cancer 1976;37:671-6.

6 Olweny CLM, Katongole-Mbidde E, Kaddu-Mukasa A, et al. Treatment of Burkitt's lymphoma: randomized clinical trial of single-agent versus combination chemotherapy. Int $J$ Cancer 1976;17:436-40.

${ }^{7}$ Ladjadj Y, Philip T, Lenoir GM, et al. Abdominal Burkitt-type lymphomas in Algeria. Br J Cancer 1984;49:503-12.

${ }^{8}$ Murphy SB. Childhood non-Hodgkin's lymphoma. $N$ Engl J Med 1978;299:1446-8.

9 Wollner N, Exelby PR, Lieberman PH. Non-Hodgkin's lymphoma in children. Cancer 1979;44:1990-9.

${ }^{10}$ Carabell SC, Cassady JR, Weinstein HJ, et al. The role of radiation therapy in the treatment of pediatric non-Hodgkin's lymphomas. Cancer 1978;42:2193-205.

11 Murphy SB, Hustu HO. A randomized trial of combined modality therapy of childhood non-Hodgkin's lymphoma. Cancer 1980;45:630-7.

12 Mott MG, Eden OB, Palmer MK. Adjuvant low dose radiation in childhood non-Hodgkin's lymphoma. $\mathrm{Br} J$ Cancer 1984:50:463-9.

13 Applebaum FR, Deisseroth AB, Graw RG, et al. Prolonged complete remission following high dose chemotherapy of Burkitt's lymphoma in relapse. Cancer 1978;41:1059-63.

14 Philip T, Biron P, Maraninchi D, et al. Role of massive chemotherapy and autologous bone-marrow transplantation in non-Hodgkin's malignant lymphoma. Lancet 1984;i:391.

15 Bleyer WA, Coccia PF, Sather HN, et al. Reduction in central nervous system leukemia with a pharmacokinetically derived intrathecal methotrexate dosage regimen. Journal of Clinical Oncology 1983;1:317-25.

16 Lynch RE, Kjellstrand CM, Coccia PF. Renal and metabolic complications of childhood non-Hodgkin's lymphoma. Semin Oncol 1977;4:325-34.

17 Ziegler JL. Treatment results of 54 American patients with Burkitt's lymphoma are similar to the African experience. $N$ Engl J Med 1977;297:75-80.

18 Anderson JR, Wilson JF, Jenkin DT, et al. Childhood nonHodgkin's lymphoma. The results of a randomized therapeutic trial comparing a 4-drug regimen (COMP) with a 10-drug regimen $\left(\mathrm{LSA}_{2}-\mathrm{L}_{2}\right)$. $N$ Engl $J$ Med 1983;308:559-65.

19 Magrath IT, Janus C, Edwards BK, et al. An effective therapy for both undifferentiated (including Burkitt's) lymphomas and lymphoblastic lymphomas in children and young adults. Blood 1984;63:1102-11.

20 Sullivan MP, Ramirez I. Curability of Burkitt's lymphoma with high-dose cyclophosphamide-high-dose methotrexate therapy and intrathecal chemoprophylaxis. Journal of Clinical Oncology 1985;3:627-36.

21 Nkrumah FK, Biggar RJ, Neequaye J. Chemotherapy of Burkitt's lymphoma: randomized clinical trial of single agent versus combination chemotherapy. West African Journal of Medicine 1983;2:9-12.

Correspondence to Dr J Pritchard, Department of Haematology and Oncology, The Hospital for Sick Children, Great Ormond Street, London WC1N 3JH.

Received 5 June 1986 